American Journal of Applied Sciences 4 (3): 176-180, 2007

ISSN 1546-9239

(C) 2007 Science Publications

\title{
Tracking Error Analysis of a Rotation-Elevation Mode Heliostat
}

\author{
Omar Aliman, Ismail Daut, Muzamir Isa, Mohd Rafi Adzman \\ School of Electrical System Engineering, Kolej University Kejuruteraan Utara Malaysia (KUKUM) \\ 02600 Jejawi, Perlis, Malaysia
}

\begin{abstract}
For the past few years, great efforts have been done in improving the tracking accuracy of a newly proposed rotation-elevation tracking mode heliostat. A special simulation program has been developed to systematically analyze the image movement and to find out the error of the parameters. In the simulation program, ray-tracing method was applied to work out the central point position of the master mirror image on the target plane during the primary tracking. From the experiment, less than $5 \mathrm{~cm}$ of tracking error was achieved with the help of the simulation program. We discussed the error analysis of the two prototypes of so called Non-Imaging Focusing Heliostat (NIFH) in University Teknologi Malaysia (UTM) which has greatly reduced the optical alignment process and resulting more precise result.
\end{abstract}

Key words: Ray tracing, heliostat, optical alignment, solar energy

\section{INTRODUCTION}

The two prototypes of $\mathrm{NIFH}^{[1]}$ built in UTM are structurally close-loop. The feedback signal from the optical encoder will eliminate the tracking error due to the mechanical lashes, wind effect and other disturbances to the heliostat frame. The pointing error existed in the tracking is basically due to the error of the parameters (i.e. the orientation angles of the rotation axis respect to the target, the latitude, etc.) and the imperfection of mechanical design. The imperfections of the mechanical design in the heliostat structure are the translation offset of reflector from the elevation axis, the mechanical alignment between the elevation axis and the rotation axis etc. In order to determine the accurate parameter, systematic analysis of the image movement has been performed using computer simulation.

Computer simulation: The primary tracking error analysis is performed base on the simulation program developed by Chong ${ }^{[2]}$. In the analysis, only a single beam that strikes on the central point of the master mirror is traced. The flow chart of the algorithm for computing and plotting the pattern of master mirror image movement from time to time in the daily sun tracking is illustrated in Fig. 1. In the beginning of the program, the user must enter the measured parameters (which we will use to calculate the heliostat orientation angles for the sun tracking), the actual parameters (which will cause the tracking error), the common parameters, the error of rotation angle $(\delta \rho)$ and the error of elevation angle $(\delta \theta)$. The measured parameters and actual parameters include facing angle ( $\varphi$ and $\left.\varphi^{\prime}\right)$, target angle ( $\lambda$ and $\left.\lambda^{\prime}\right)$, latitude ( $\phi$ and $\phi^{\prime}$ ) and number of the day (NOD and NOD'). The common parameters that needed in the simulation are such as target distance (L), longitudinal correction (LongC), offset distance of the reflector from the elevation axis (EOff), time delay of the heliostat tracking ( $\delta$ LCT) and encoder revolution (Rev). There are two options in the simulation program: one is the target orientation and the other is the correction of the reflector from the elevation axis.

In the program algorithm, the computation of the sun position angles $\left(\beta^{\prime}, \rho^{\prime}\right)$ and the heliostat orientation angles $(\theta, \rho)$ will be done first. The angles $\beta^{\prime}$ and $\rho^{\prime}$ are estimated through the equation (3.31) and (3.32) from the actual parameters such as $\phi^{\prime}, \lambda^{\prime}, \phi^{\prime}$, NOD' and LCT- $\delta$ LCT. On the other hand, the angles $\theta$ (elevation movement) and $\rho$ (rotation movement) are computed through the Eq. (1) and (2) from the measured parameters such as $\phi, \lambda, \phi$, NOD and LCT.

$\rho=\operatorname{ArcSin}\left\{\frac{-\operatorname{Cos} \delta \operatorname{Cos} \omega \operatorname{Sin} \phi \operatorname{Sin} \Phi+\operatorname{Cos} \delta \operatorname{Sin} \omega \operatorname{Cos} \phi+\operatorname{Sin} \delta \operatorname{Sin} \phi \operatorname{Cos} \Phi}{\operatorname{Cos} \beta}\right\}(1)$

Corresponding Author: Omar Aliman, School of Electrical System Engineering, Kolej University Kejuruteraan Utara Malaysia (KUKUM), 02600 Jejawi, Perlis, Malaysia, Tel: +604-9798146, Fax: +604-9798304 
$\theta=\frac{\pi}{4}-\frac{1}{2} \operatorname{ArcSin}\left\{\begin{array}{l}-\operatorname{Cos} \delta \operatorname{Cos} \omega(\operatorname{Sin} \lambda \operatorname{Cos} \Phi+\operatorname{Cos} \lambda \operatorname{Cos} \phi \operatorname{Sin} \Phi)-\operatorname{Cos} \delta \operatorname{Sin} \omega \\ \operatorname{Cos} \lambda \operatorname{Sin} \phi+\operatorname{Sin} \delta(\operatorname{Cos} \lambda \operatorname{Cos} \phi \operatorname{Cos} \Phi-\operatorname{Sin} \Phi \operatorname{Sin} \lambda)\end{array}\right\}$ (2)

Another consideration of the tracking error is the achievable precision of the heliostat orientation angles. It is limited by the encoder revolution where the smallest steps size of the angles $\theta$ and $\rho$ are equal to $2 \pi$ /Rev. From the angles $\theta, \rho, \beta^{\prime}$ and $\rho$, the central point coordinate of the master mirror, the unit vector of incidence ray, the unit vector of master mirror normal and the unit vector of reflected ray can be determined

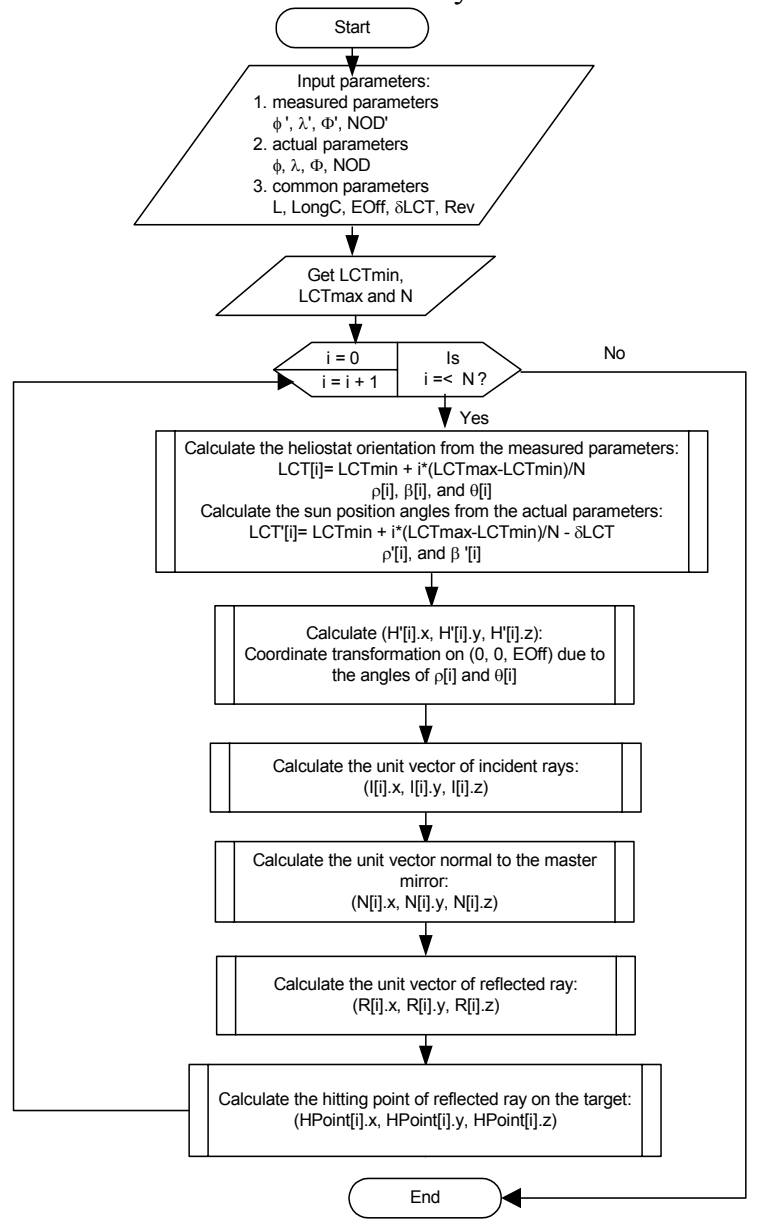

Fig. 1: Flowchart of the simulation program algorithm in primary tracking error analysis

through the Eq. (3)-(6). Finally from the Eq. (7), the hitting point of sunray at the target will be obtained. This algorithm will iterate $\mathrm{N}$ times starting from local time 0800 (LCTmin) and ending at local time 1800 (LCTmax). The following is the equations used in the simulation to obtain the central point of master mirror images on the target during the daily sun tracking:

$$
\begin{aligned}
& {\left[\begin{array}{c}
\mathrm{H}^{\prime}[\mathrm{i}] \cdot \mathrm{x} \\
\mathrm{H}^{\prime}[\mathrm{i}] \cdot \mathrm{y} \\
\mathrm{H}^{\prime}[\mathrm{i}] \cdot z
\end{array}\right]=\left[\begin{array}{c}
\text { EOff } \cos (\rho[\mathrm{i}]) \sin (\theta[\mathrm{i}]) \\
- \text { EOff } \sin (\rho[\mathrm{i}]) \sin (\theta[\mathrm{i}]) \\
\text { EOff } \cos (\theta[\mathrm{i}])
\end{array}\right]} \\
& {\left[\begin{array}{l}
\mathrm{I}[\mathrm{i}] \cdot \mathrm{x} \\
\mathrm{I}[\mathrm{i}] \cdot \mathrm{y} \\
\mathrm{I}[\mathrm{i}] \cdot \mathrm{z}
\end{array}\right]=\left[\begin{array}{c}
\cos \beta^{\prime} \cos \rho^{\prime} \\
-\cos \beta^{\prime} \sin \rho^{\prime} \\
\sin \beta^{\prime}
\end{array}\right]} \\
& {\left[\begin{array}{l}
\mathrm{N}[\mathrm{i}] \cdot \mathrm{x} \\
\mathrm{N}[\mathrm{i}] \cdot \mathrm{y} \\
\mathrm{N}[\mathrm{i}] \cdot z
\end{array}\right]=\left[\begin{array}{c}
\sin \theta \cos \rho \\
-\sin \theta^{\prime} \sin \rho \\
\cos \theta
\end{array}\right]} \\
& {\left[\begin{array}{l}
\mathrm{R}[\mathrm{i}] \cdot \mathrm{x} \\
\mathrm{R}[\mathrm{i}] \cdot \mathrm{y}
\end{array}\right]=\left[\begin{array}{l}
2(\mathrm{I}[\mathrm{i}] \cdot \mathrm{xN}[\mathrm{i}] \cdot \mathrm{x}+\mathrm{I}[\mathrm{i}] \cdot \mathrm{yN}[\mathrm{i}] \cdot \mathrm{y}+\mathrm{I}[\mathrm{i}] \cdot \mathrm{zN}[\mathrm{i}] \cdot \mathrm{z}) \mathrm{N}[\mathrm{i}] \cdot \mathrm{x}-\mathrm{I}[\mathrm{i}] \cdot \mathrm{x} \\
2(\mathrm{I}[\mathrm{i}] \mathrm{xN}[\mathrm{i}] \cdot \mathrm{x}+\mathrm{I}[\mathrm{i}] \cdot \mathrm{yN}[\mathrm{i}] \cdot \mathrm{y}+\mathrm{I}[\mathrm{i}] \cdot \mathrm{zN}[\mathrm{i}] \mathrm{z}) \mathrm{N}[\mathrm{i}] \mathrm{x}-\mathrm{I}[\mathrm{i}] \mathrm{y}
\end{array}\right.} \\
& R[i] \cdot y=2(I[i] \cdot x N[i] \cdot x+I[i] \cdot y N[i] \cdot y+I[i] \cdot z N[i] \cdot z) N[i] \cdot x-I[i] \cdot y \\
& \text { R[i].z } \quad 2(I[i] \cdot x N[i] \cdot x+I[i] \cdot y N[i] \cdot y+I[i] \cdot z N[i] \cdot z) N[i] \cdot x-I[i] \cdot z] \\
& {\left[\begin{array}{l}
\text { HPoint }[\mathrm{i}] \cdot \mathrm{x} \\
\mathrm{HPoint}[\mathrm{i}] \cdot \mathrm{y} \\
\mathrm{HPoint}[\mathrm{i}] \cdot z
\end{array}\right]=\left[\begin{array}{l}
\mathrm{R}[\mathrm{i}] \cdot \mathrm{x}\left(\mathrm{L}-\mathrm{H}^{\prime}[\mathrm{i}] \cdot \mathrm{z}\right) / \mathrm{R}[\mathrm{i}] \cdot \mathrm{z}+\mathrm{H}^{\prime}[\mathrm{i}] \cdot \mathrm{x} \\
\mathrm{R}[\mathrm{i}] \cdot \mathrm{y}\left(\mathrm{L}-\mathrm{H}^{\prime}[\mathrm{i}] \cdot \mathrm{z}\right) / \mathrm{R}[\mathrm{i}] \cdot \mathrm{z}+\mathrm{H}^{\prime}[\mathrm{i}] \cdot \mathrm{y} \\
\mathrm{R}[\mathrm{i}] \cdot z\left(\mathrm{~L}-\mathrm{H}^{\prime}[\mathrm{i}] \cdot \mathrm{z}\right) / \mathrm{R}[\mathrm{i}] \cdot \mathrm{z}+\mathrm{H}^{\prime}[\mathrm{i}] \cdot z
\end{array}\right] \text { (7) }}
\end{aligned}
$$

Tracking error analysis: Simulation program analysis on the tracking error study has been adopted in the first prototype (with the target distance of $10 \mathrm{~m}$ ) and second prototype (with the target distance of $16.5 \mathrm{~m}$ ) of NIFH heliostat. Since the optical encoders used in the system are incremental type, the initial values of elevation angle $\theta$ and rotation angle $\rho$ have to be provided. These initial values are measured using slant level $\left(90^{\circ}\right.$ range, $0.5^{\circ}$ error). Normally, in order to reduce the measurement error we normally initialize the heliostat to the orientation at solar noon in which the arm is horizontal.

The computer program had no way to determine the absolute position of the heliostat since incremental optical encoders were applied in the control system. Even though the last position of heliostat can always be recorded for reference in the next operating schedule, there is no guarantee that heliostat position will not change due to external disturbances when computer is turned off. Therefore, prior to a new tracking session, the initial position of heliostat, the rotation and elevation of master mirror, were manually measured and entered into the program. Then, the starting time of a tracking session was set.

Throughout the experiment, this method can achieve a reasonable good accuracy in sun tracking. One of the major errors that cannot be avoided in the measurement of facing angle is the deviation between the direction of true north and the magnetic north. However, the precision of the facing angle, $\phi$ and target angle, $\lambda$ measurement can be further corrected through the tracking error analysis study using simulation program.

\section{RESULTS}

First prototype: In order to determine the actual parameter, the simulated tracking error pattern is compared with the recorded tracking error pattern. In the experiment, the measured facing angle and target 
angle are estimated to be $0^{\circ}$ and $0^{\circ}$ respectively. During the daily sun tracking, the image of the master mirror on the target is recorded using CCD camera from 0836 hour to 1815 hour on August 18 as shown in Fig. 2. From the recorded tracking error pattern, the actual parameters can be analyzed and predicted through the simulation program. In the simulation, the actual facing angle $\phi^{\prime}$ is $0.2^{\circ}$, target angle $\lambda^{\prime}$ is $0.1^{\circ}$, measurement error of elevation angle $\delta \theta$ is estimated as $0.02^{\circ}$ and rotation angle $\delta \rho$ is $0.15^{\circ}$.

On the next day, the sun tracking are performed from 0912 hour to 1730 hour with the predicted actual parameters, e.g. the facing angle is $0.2^{\circ}$ and the target is $0.1^{\circ}$. With this setting, the longest distance between two master images is less than $2.5 \mathrm{~cm}$ for 8 hours of sun tracking.

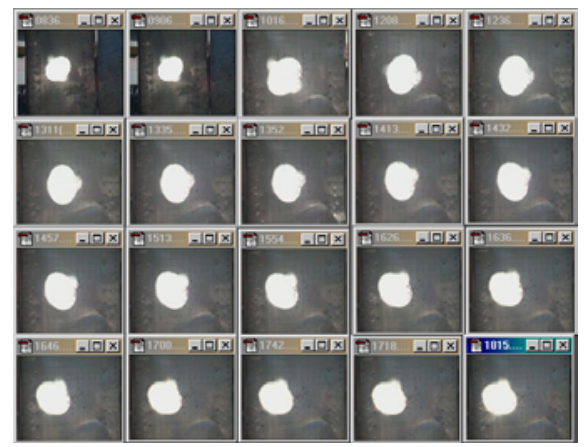

Fig. 2: Observation on August 18, 2005

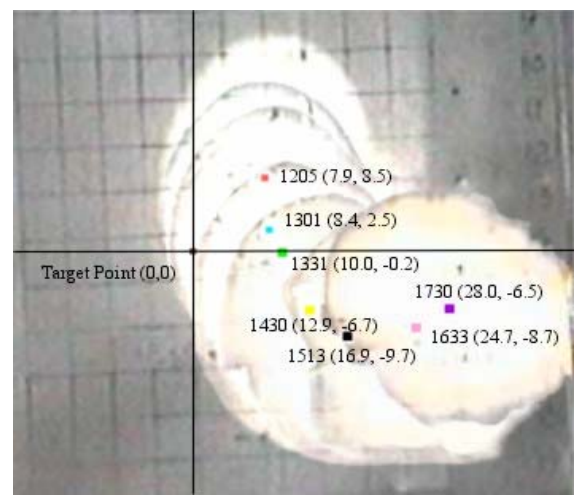

Fig. 3: The recorded result of the tracking error pattern on July 13

Another experiment that was conducted by Chong $^{[2]}$, the measured facing angle and target angle are estimated as $-0.6^{\circ}$ and $0^{\circ}$ respectively. During the daily sun tracking, the image of the master mirror on the target is recorded using CCD camera (Fig. 3) from 1205 hour to 1730 hour on July 13. From the recorded tracking error pattern, the nearest tracking error pattern of the simulated result to the experimental result are shown as Fig. 4. In the simulation, the actual facing angle $\phi^{\prime}$ is $1.2^{\circ}$, target angle $\lambda^{\prime}$ is $0^{\circ}$, measurement error of elevation angle $\delta \theta$ is estimated as $0.01^{\circ}$ and rotation angle $\delta \rho$ is $0.15^{\circ}$.

On the next day, the sun tracking are performed from 1330 hour to 1802 hour with the predicted actual parameters, e.g. the facing angle is $1.2^{\circ}$ and the target angle is $0^{\circ}$. With this setting, the longest distance between two master images is less than $2.5 \mathrm{~cm}$ for $4 \frac{1}{2}$ hours of sun tracking (Fig. 5).

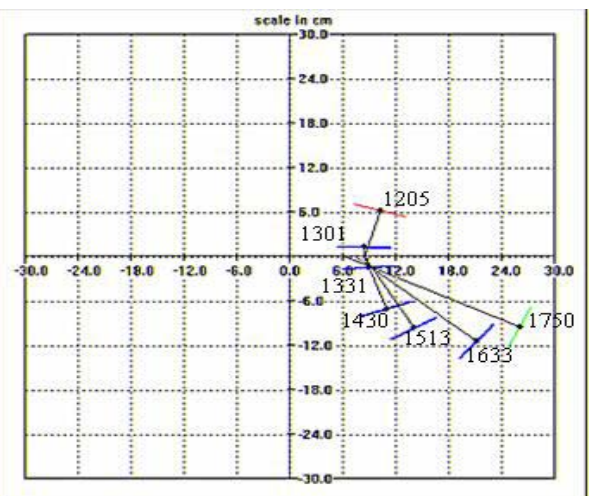

Fig. 4: The simulation result of the daily tracking error pattern

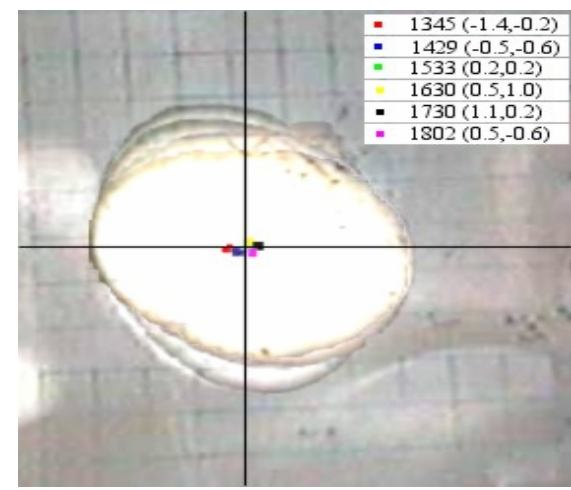

Fig. 5: The pattern of sun tracking from 1330 hour to 1802 hour on 14 July with the predicted actual parameters

Second prototype: The measured facing angle and target angle are estimated as $187^{\circ}$ and $-5.5^{\circ}$ respectively. During the daily sun tracking, the image of the master mirror on the target is recorded using CCD camera from 0930 hour to 1500 hour on September 15 as shown in Fig. 6. However this parameter gave an error of $65 \mathrm{~cm}$ from the exact target point. The recorded tracking pattern has been analyzed and predicted through the simulation program. The nearest tracking error pattern of the simulated result to the experimental result are shown as Fig. 7. In the simulation, the actual facing angle $\phi^{\prime}$ is $183.5^{\circ}$, target angle $\lambda^{\prime}$ is $-4.6^{\circ}$. 
Table 1: Example data of the master mirror offset

\begin{tabular}{|c|c|c|c|c|}
\hline Solar Time & Incidence Angle & New Inc. Angle & Different Angle & Error $(\mathrm{mm})$ \\
\hline 8.00 & 50.82392 & 50.38256 & -0.44136 & 38.7604 \\
\hline 8.30 & 50.87619 & 50.43450 & -0.44169 & 38.7892 \\
\hline 9.00 & 50.98701 & 50.54461 & -0.44239 & 38.8501 \\
\hline 9.30 & 51.02901 & 50.58635 & -0.44266 & 38.8732 \\
\hline 10.00 & 51.11228 & 50.66910 & -0.44318 & 38.9188 \\
\hline 10.30 & 51.14112 & 50.69775 & -0.44336 & 38.9346 \\
\hline 11.00 & 51.19110 & 50.74741 & -0.44368 & 38.9620 \\
\hline 11.30 & 51.20477 & 50.76100 & -0.44376 & 38.9695 \\
\hline 12.00 & 51.21799 & 50.77414 & -0.44385 & 38.9767 \\
\hline 12.30 & 51.21555 & 50.77172 & -0.44383 & 38.9754 \\
\hline 13.00 & 51.19118 & 50.74741 & -0.44368 & 38.9620 \\
\hline 13.30 & 51.17272 & 50.72916 & -0.44356 & 38.9519 \\
\hline 14.00 & 51.11228 & 50.66910 & -0.44318 & 38.9188 \\
\hline 14.30 & 51.07924 & 50.63627 & -0.44297 & 38.90073 \\
\hline 15.00 & 50.98701 & 50.54461 & -0.44239 & 38.85016 \\
\hline 15.30 & 50.94159 & 50.49948 & -0.44210 & 38.82521 \\
\hline 16.00 & 50.82392 & 50.38256 & -0.44136 & 38.76049 \\
\hline 16.30 & 50.76925 & 50.32823 & -0.44101 & 38.73026 \\
\hline 17.00 & 50.63425 & 50.19409 & -0.44016 & 38.65564 \\
\hline 17.30 & 50.57407 & 50.13429 & -0.43977 & 38.62231 \\
\hline 18.00 & 50.43097 & 49.99211 & -0.43886 & 38.54288 \\
\hline
\end{tabular}

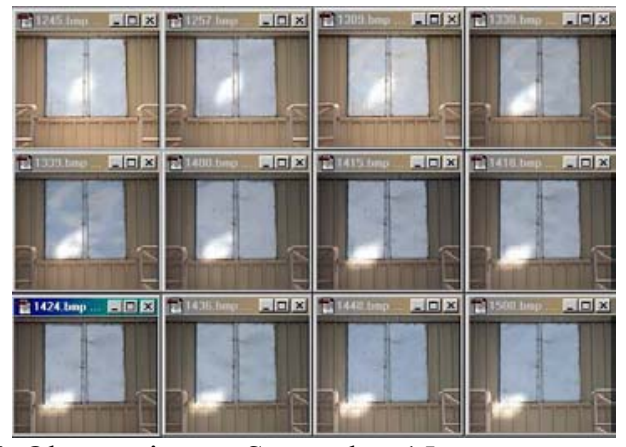

Fig. 6: Observation on September 15

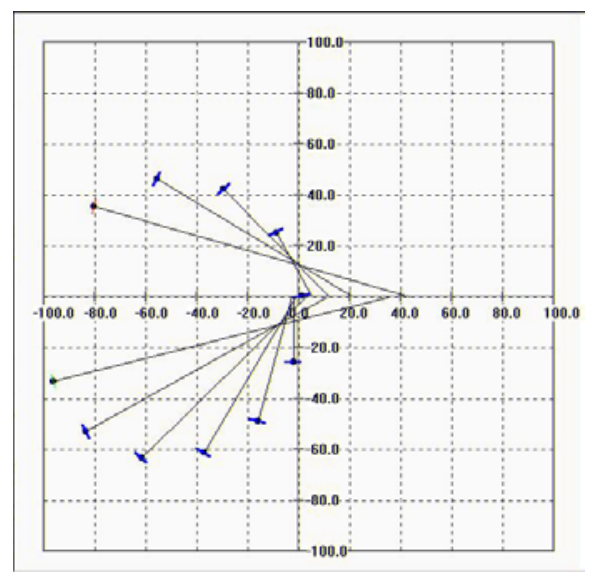

Fig. 7: The simulation result of the daily tracking error pattern on September 15

Finally, three days later, the sun tracking are performed from 0924 hour to 1621 hour with the

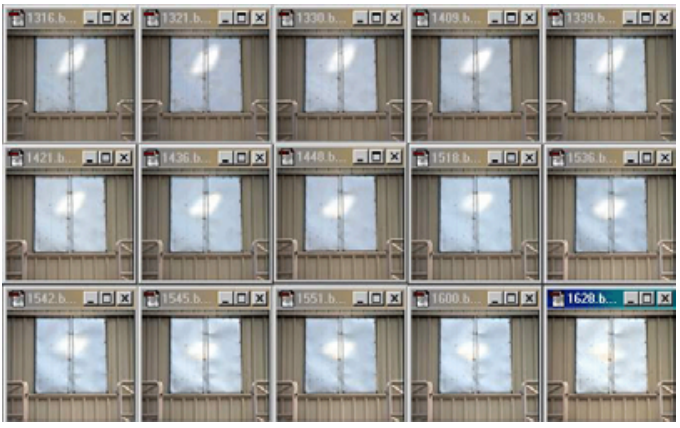

Fig. 8: Image tracking after correction

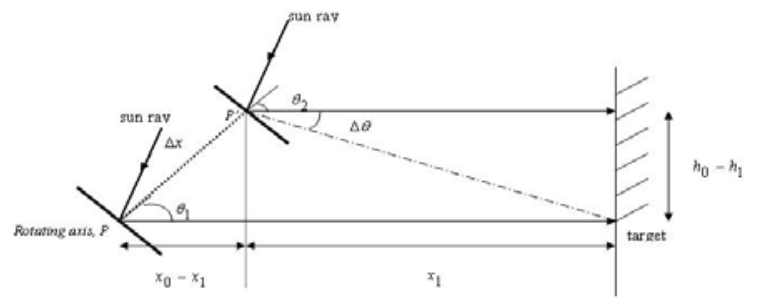

Fig. 9: Master mirror offset

predicted actual parameters, e.g. the facing angle is $180^{\circ}$, target angle $\lambda^{\prime}$ is $-3.7^{\circ}$ and the result is shown in Fig. 8. With this setting, the longest distance between two master images is less than $4.5 \mathrm{~cm}$ for 8 hours of sun tracking.

Master mirror offset: The master mirror of the heliostat that acts as a reference for sun tracking will constantly focus to a stationary target as illustrated in 
Fig. 9. The center of the master mirror should be placed exactly at the rotating axis point, $\mathrm{P}$. But, if there is an offset, $\Delta x$ from the rotating axis, $P$ and the center point of the master mirror, $\mathrm{P}^{\prime}$, the incidence angle, $\theta$ should be corrected as,

$$
\theta_{\text {new }}=\theta+\Delta \theta
$$

where, $\Delta \theta$ is the correction of the incidence angle

$$
\Delta \theta=\tan ^{-1}\left(\frac{\Delta \mathrm{x} \sin \theta}{\mathrm{x}_{0}-\Delta \mathrm{x} \cos \theta}\right)
$$

Table 1 illustrates an example of the data taken on August 19, using the first prototype of NIFH. For an offset of $0.5 \mathrm{~cm}$, the image distance from the ideal position is about $39 \mathrm{~mm}$. Obviously, the image will not focus to a stationary target. The longer the distance between the heliostat and the target, the bigger error will be made.

\section{ACKNOWLEDGMENT}

We acknowledge with many thanks to Prof. Y.T.Chen and members of Solar Group which we formerly in the same team and effort, together struggling for the success of this research while we were in Universiti Teknologi Malaysia.

\section{CONCLUSION}

From the experiment, both prototypes of the NIFH heliostat achieved less than $5 \mathrm{~cm}$ tracking error in average. The factors of master mirror offset, the accuracy of the measurement and high resolution of the positioning device have been identified to contribute errors. New calculation of the incidence angle, new technique to make alignment and high performance device has been implemented to reduce the problems. The introductions of the optical alignment methods have significantly reduced the time and manpower cost. Due to the uncertainty of the structural rigidity upon installation of the frame at the arm of the heliostat, further alignment work has to be done to confirm the rotational axis of the heliostat frame.

\section{REFERENCES}

1. Chen, Y.T., K.K. Chong, O. Aliman, T.P. Bligh1, L.C. Chen, J. Yunus, K.S. Kannan, B.H. Lim, C.S. Lim, M.A. Alias, N. Bidin, S. Salehan, S.A. Rezan S.A.H., C.M. Tam and K.K. Tan, 2001. Nonimaging focusing heliostat. Intl. J. Solar Energy, 71: 155-164.

2. Chong, K.K., 2002. Non-imaging focusing heliostat. $\mathrm{Ph}$. D. Thesis, Universiti Teknologi Malaysia, Skudai. 